\title{
Astronomical $\begin{gathered}\text { Joural } \\ \text { Telescopes, }\end{gathered}$ Instruments, and Systems
}

AstronomicalTelescopes.SPIEDigitalLibrary.org

\section{Vibration budget for observatory equipment}

Douglas G. MacMartin

Hugh Thompson

\section{SPIE.}




\title{
Vibration budget for observatory equipment
}

\author{
Douglas G. MacMartin a,b,* and Hugh Thompson ${ }^{\mathrm{b}}$ \\ ${ }^{a}$ California Institute of Technology, Computing and Mathematical Sciences, 1200 East California Boulevard, Pasadena, California 91125, \\ United States

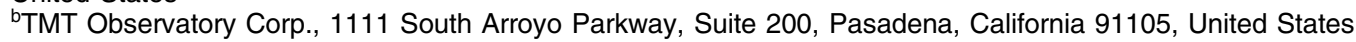

\begin{abstract}
Vibration from equipment mounted on the telescope and in summit support buildings has been a source of performance degradation at existing astronomical observatories, particularly for adaptive optics performance. Rather than relying only on best practices to minimize vibration, we present here a vibration budget that specifies allowable force levels from each source of vibration in the observatory (e.g., pumps, chillers, cryocoolers, etc.). This design tool helps ensure that the total optical performance degradation due to vibration is less than the corresponding error budget allocation and is also useful in design trade-offs, specifying isolation requirements for equipment, and tightening or widening individual equipment vibration specifications as necessary. The vibration budget relies on model-based analysis of the optical consequences that result from forces applied at different locations and frequencies, including both image jitter and primary mirror segment motion. We develop this tool here for the Thirty Meter Telescope but hope that this approach will be broadly useful to other observatories, not only in the design phase, but for verification and operations as well. ๑ 2015 Society of Photo-Optical Instrumentation Engineers (SPIE) [DOI: 10.1117/1.JATIS.1.3.034005]
\end{abstract}

Keywords: vibration; extremely large telescopes; performance; adaptive optics.

Paper 15017P received Apr. 2, 2015; accepted for publication Aug. 4, 2015; published online Sep. 16, 2015.

\section{Introduction}

Vibration due to equipment both on and off the telescope is a source of potential performance degradation, particularly for adaptive optics (AO) observations. Vibration impacts performance at most operating observatories; see, for example, the comprehensive review by Kulcsár et al. ${ }^{1}$ - this issue has been a challenge for many years. ${ }^{2}$ For the Thirty Meter Telescope (TMT), the error budget allocates $30 \mathrm{~nm}$ for the residual AOcorrected rms wavefront error (WFE) due to all vibration sources, including WFEs due to both image jitter (IJ) and M1 segment motion. This is significantly better than what is typically achieved at existing observatories and will require careful attention during final design and construction.

Efforts are being made to reduce the impact of vibration on the delivered AO performance by changing the AO control algorithm ${ }^{3-5}$ without changing the vibration environment; similar approaches are being explored for interferometer optical-pathdifference control. ${ }^{6}$ Passive or active damping can also be used to change the structural response to vibration; ${ }^{7}$ this changes the vibration environment without changing the sources of vibration. And isolation or source relocation can be used to directly modify source levels. ${ }^{8}$ All of these are important components of an overall strategy to reach an acceptable and cost-effective solution. However, during the design phase, it is invaluable to have a systematic design tool that can be used to levy requirements on subsystems, allow design trades, and track status relative to the top-level performance requirement. Tracking performance with an error budget is standard practice in any observatory design; this vibration budget serves a similar role.

\footnotetext{
*Address all correspondence to: Douglas G. MacMartin, E-mail: macmardg@
} caltech.edu
To ensure that TMT will deliver acceptable AO image quality despite equipment vibration, we thus place requirements on source amplitudes (in Newtons) for every significant potential source of vibration. This, in turn, will affect design decisions such as locating sources off telescope where possible, selecting well-balanced low-vibration equipment, and defining isolation requirements. This design tool will also be used during acceptance and verification of subsystems to ensure that the vibration environment of the telescope is acceptable as built and minimize the potential for costly diagnostics and fixes during operations. Furthermore, we anticipate that the vibration budget will be maintained for the lifetime of the observatory both to ensure that new equipment meets requirements and to aid in diagnosis should the vibration environment change.

Our basic approach is to (1) use the telescope finite element model (FEM) to evaluate the optical sensitivity to forces applied at different locations and at different frequencies, (2) use this sensitivity analysis to place requirements on all significant sources of vibration so that if these subsystem requirements are met, the vibration contribution to the error budget is met, and (3) evaluate potential vibration sources to determine what steps (e.g., isolation) would be needed to meet these requirements. We intend to complement this model-based approach with transfer-function measurements made using calibrated sources as the observatory is built; initial measurements at Subaru Observatory are described in Ref. 9. Improved estimates for force levels from different sources will be used to reallocate force budgets to achieve the most cost-effective solution.

Note that in many applications, the vibration environment is specified in terms of the power spectrum of acceleration.

$2329-4124 / 2015 / \$ 25.00$ (C) 2015 SPIE 
However, while this is appropriate to determine, for example, isolator requirements for some sensitive instrument that is mounted in such an environment, it is not sufficient to ensure that such an acceleration power spectrum is met, which requires placing force requirements on sources.

The key analysis here is the modeling of optical sensitivity as described in Sec. 2, including contributions from both image jitter (tip/tilt) and higher-order terms due to motion of the 492 primary mirror segments at TMT; preliminary results can also be found in Refs. 10 and 11. The current vibration budget is given in Table 2 in Sec. 3. The specification applies to the rms force level in Newtons for each source after passing through a filter that has a unit gain from 5 to $20 \mathrm{~Hz}$, but falls off above and below this frequency range. This allows for higher forces at either higher or lower frequencies where the AO performance is less sensitive; the shape of the filter is motivated from the modeling described in Sec. 2. Note that many of the details given here are specific to TMT, including some of the source locations selected for analysis, the quantitative results for the sensitivity to vibration at those locations, and the chosen allocation of forces that make up the budget. However, we present the vibration budget here because we believe that this new methodology will be valuable to other observatories, particularly during the design and construction phases, but potentially during operations as well.

\section{Sensitivity Analysis}

We consider the following input locations for vibration forces, also illustrated in Fig. 1. This is clearly not a complete list, but captures most of the larger sources of potential vibration, and gives a representative collection to illustrate the variation in sensitivity to forces at different locations.

1. Pier forces (applied uniformly at the base of the azimuth track): Note that all off-telescope sources affect the optical response through motion of the pier; the propagation of forces from the enclosure or facilities building is not included in the FEM but is captured by an estimated attenuation factor anchored from data taken at Subaru Observatory ${ }^{9}$ (see Fig. 7).

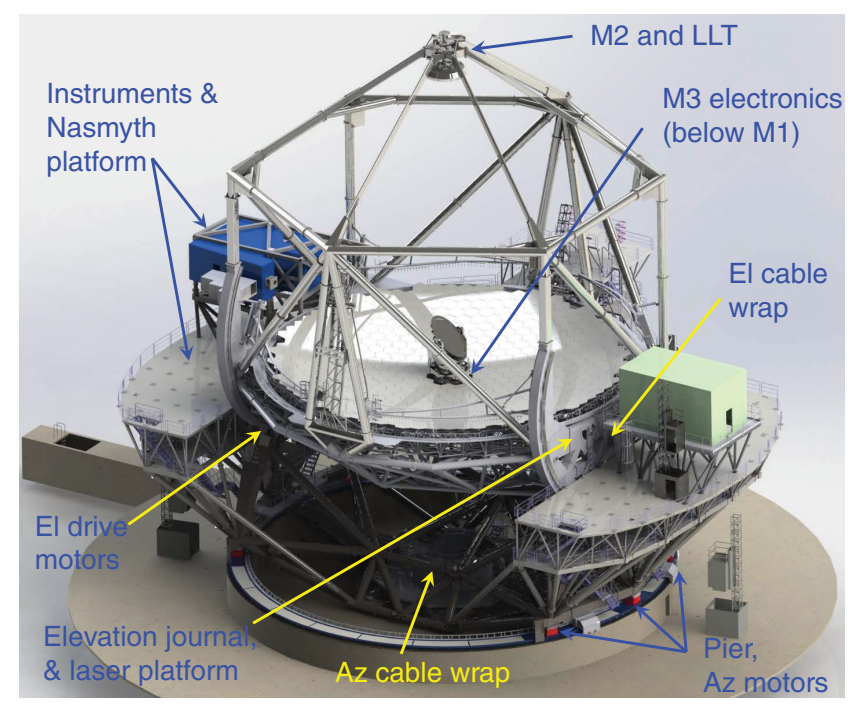

Fig. 1 Force input locations considered for evaluating optical sensitivity to vibration for the Thirty Meter Telescope (TMT).
2. Instruments and other sources on the Nasmyth platforms: Input forces have been considered at all instrument mass nodes and on the Nasmyth platform itself; the response is similar and results are shown here only for forces at one instrument (MIRES).

3. Azimuth and elevation cable wraps: These are modeled as torques about the respective drive axes. For consistency with other inputs, the response is given per Newton of force, using the cable-wrap radius to scale the moment arm.

4. Laser launch telescope (LLT) and other sources near M2.

5. Sources mounted on the elevation journals: For TMT, this includes M2 electronics and the lasers; the latter are mounted on the elevation journal with electronics mounted on a platform attached to the journal.

6. M3 electronics, at the base of the M3 tower: M1 electronics boxes are distributed under the primary mirror, but the optical sensitivity is assumed to be similar to the central location where M3 electronics are placed.

7. Elevation axis direct drive motor on the azimuth structure.

8. Azimuth axis direct drive motor: These forces affect the system similar to pier forces with one caveat noted below.

The last five locations are potential sources of vibration through the use of cooling fluid, which may exert forces from fluid turbulence that may be difficult to isolate. Vibration forces may also enter the structure at mounting

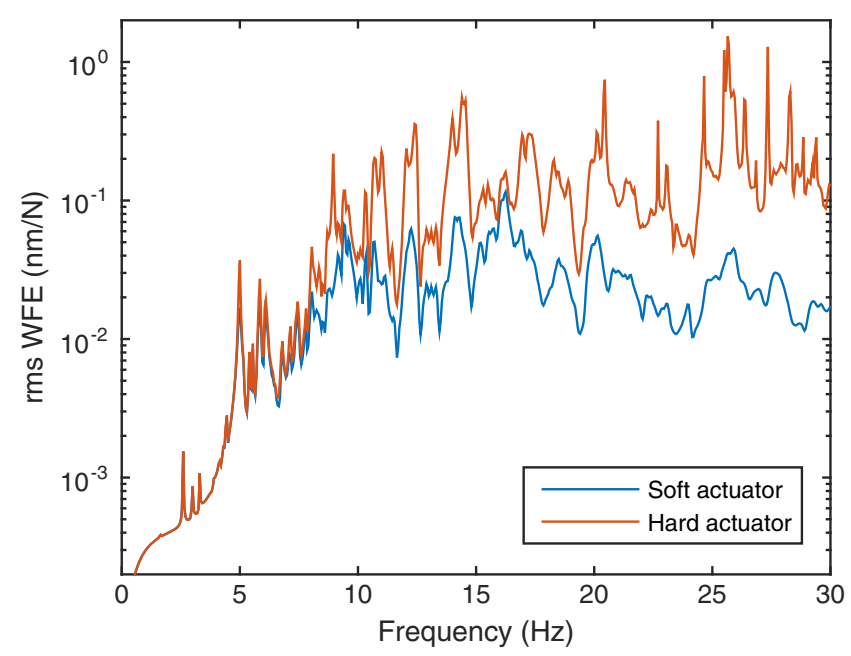

Fig. 2 Comparison of the adaptive optics (AO) corrected rms wavefront error (WFE) due to the M1 segment motion that results from vertical forcing at the telescope pier if the primary mirror control system (M1CS) actuators were hard (e.g., piezoelectric) or soft (voice-coil, TMT baseline); both the spatial and temporal correctability are included. Above the 8 to $10 \mathrm{~Hz}$ bandwidth of the actuator servo loop, the soft actuators both isolate the mirror surface from the motion of the mirror cell and provide damping; at $30 \mathrm{~Hz}$, the reduction relative to an undamped hard actuator is more than a factor of 10 . 
(a)

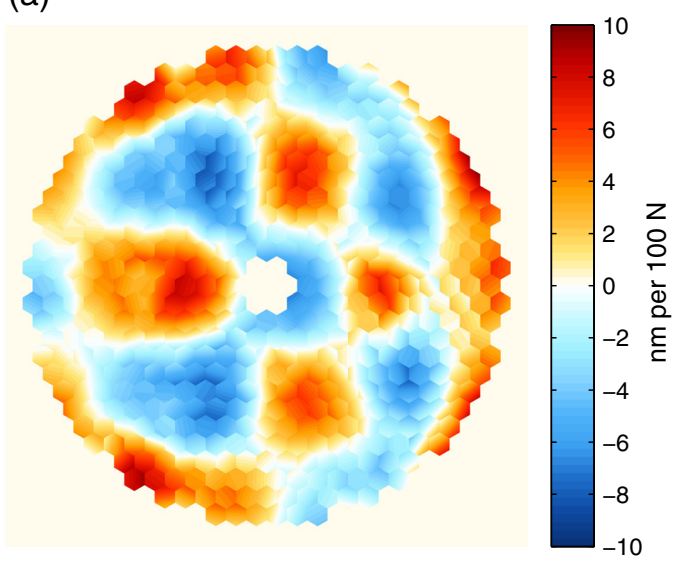

(b)

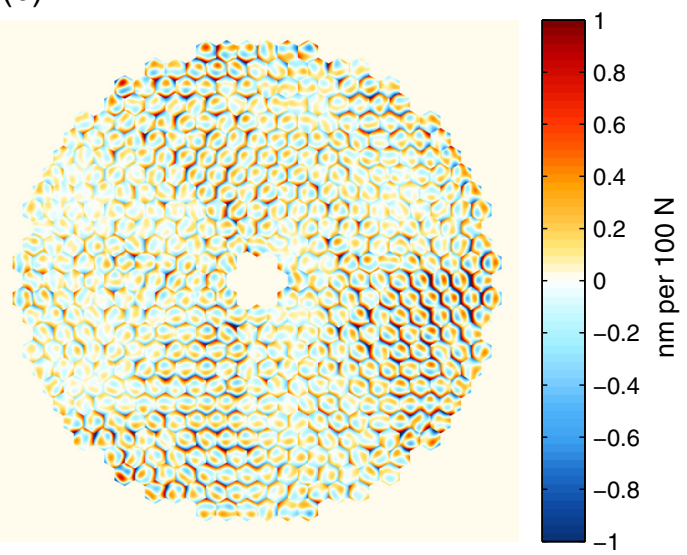

Fig. 3 Representative pattern of M1 segment motion at $30 \mathrm{~Hz}$ (a) without and (b) with AO spatial correction; note the different scale. The latter case includes only quasistatic spatial fitting errors (as if the temporal bandwidth were infinite) to illustrate that the spatial pattern of WFE is well corrected by $A O$, and correction is limited primarily by the temporal $\mathrm{AO}$ bandwidth.

brackets for the pipes carrying cooling fluid (the flow may be turbulent or include pressure fluctuations from compressors/ pumps); as the routing of these has not been established, the vibration budget uses sensitivities from representative locations in the list above. For each source location other than cable wrap torques and the telescope pier, the response is calculated for forces in each axis $\left(F_{x}, F_{y}\right.$, and $\left.F_{z}\right)$ and the sensitivities shown in Fig. 6 are calculated as the rms over all three axes. Sources off the telescope are typically isolated and create only vertical forces; these excite the pier predominantly through the propagation of surface Rayleigh waves with the resultant vertical motion roughly $50 \%$ higher than the horizontal. ${ }^{12}$ To capture this effect, we weight the vertical direction $50 \%$ higher relative to the horizontal in computing the sensitivity to pier and off-telescope forces, but use an equal weighting to compute the sensitivity to forces at the azimuth drive itself.

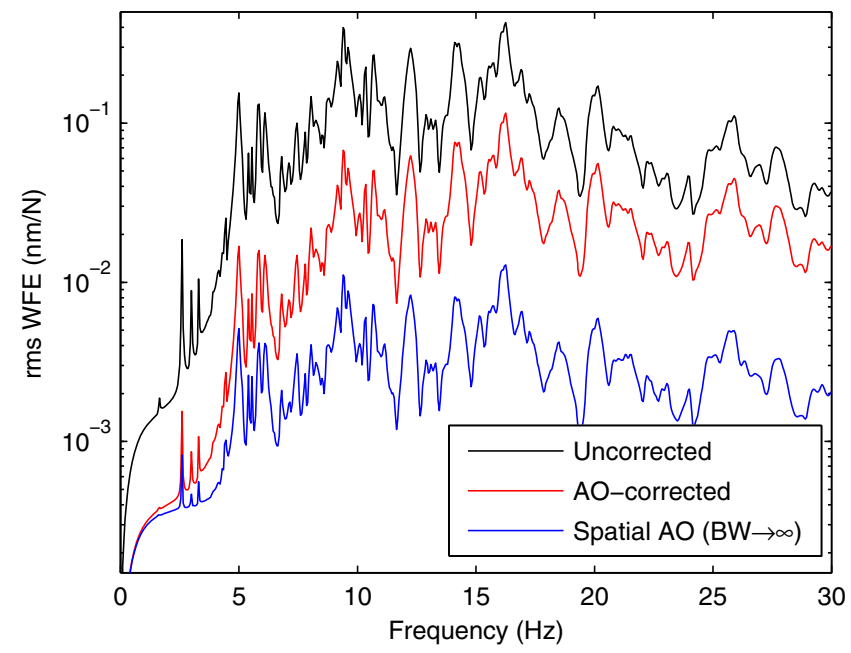

Fig. 4 WFE from $\mathrm{M} 1$ segment motion in response to $1 \mathrm{~N}$ vertical force on the pier before and after $\mathrm{AO}$ correction; also shown is the spatial correctability (as if the temporal bandwidth were infinite); consistent with Fig. 3, this illustrates that the response pattern is spatially well corrected, but correction is limited by the temporal bandwidth.
Vibration caused by sources such as bearing roughness, torque ripple, or other actuator noise within the M2 or M3 subsystems overwhelmingly results in local motion of the respective optical surface rather than vibration propagation through the structure. ${ }^{11}$ These disturbances apply equal and opposite torques to the telescope structure and the relevant optic. While the former can result in excitation of telescope resonances and motion of other optical surfaces, the resulting image jitter and M1 response are negligible compared with the direct response of the driven surface, which can be roughly estimated from the mirror inertial response. Thus, there is no need to consider these inputs with the full structural model. For TMT, these sources are separately categorized in the error budget as control noise and are directly managed by the relevant subsystem.

Forces on the telescope structure and pier result in both image jitter and M1 segment dynamic motion. We predict these effects using two separate models, since different fidelity is required.

The image jitter prediction combines the telescope FEM with a linear optical model and the mount control system (which only affects results at low frequencies). Structural damping of $0.5 \%$ is assumed, and the first 4000 modes of the telescope structure are included (up to $50 \mathrm{~Hz}$ ). A $15 \mathrm{~Hz}$ type II rejection is included for simulating the residual AO-corrected WFE from telescope image motion; the relatively low bandwidth results from the requirement of high sky coverage, combined with the need for natural guide stars for tip/tilt estimation. Seeing-limited performance is also calculated. TMT uses the normalized point-source sensitivity (PSSN) related to rms image jitter $\theta_{\mathrm{rms}}$ as PSSN $=1-\alpha \theta_{\mathrm{rms}}^{2}$, where $\alpha=9.12 \times 10^{-6}$ for $\theta_{\text {rms }}$ in milli-arcseconds.

Modeling to predict M1 segment motion additionally includes a low-order model of the segment dynamics for all 492 primary mirror segments, including the stiffness and internal resonances of the segment support assembly, actuator dynamics that include the actuator servo characteristics, and the global control loop that uses feedback of edge-sensors to maintain segment positions. This model and the control loops are described in more detail in Ref. 13. Note that TMT has selected (soft) voice-coil actuators for control of M1 segment motion, with stiffness at low frequencies provided by an $\sim 8 \mathrm{~Hz}$ servo loop. ${ }^{13}$ Above this frequency, these actuators 
serve to isolate segment motion from mirror cell motion, greatly reducing the contribution of $\mathrm{M} 1$ segment motion to the $\mathrm{AO}$-corrected WFE, as illustrated in Fig. 2. It is this improvement that drove the selection of soft actuators. Furthermore, the rate-feedback gain of the actuator servo loop, combined with a passive damping element in the TMT design, results in damping of structural resonances in addition to the isolation-based reduction in the average response. Again, both AO-corrected rms WFE and PSSN for seeing-limited observations are computed from the M1 segment motion.

The WFE from segment motion is partially corrected by the deformable mirrors (DMs) of the AO system, limited by both the finite temporal bandwidth and spatial fitting errors. These are separately computed here. A $60 \mathrm{~Hz}$ bandwidth $\left(f_{c}\right)$ is used for temporal correction of M1 segment motion by the AO system; at frequency $f$, the temporal rejection multiplies the spatially correctable pattern by $S(f)=f / \sqrt{f^{2}+f_{c}^{2}}$, where $S=1$ yields no correction and $S=0$ perfect correction. The spatial fitting error is similarly described by $A(f)$; this is the residual fraction of the amplitude that would remain if the spatial pattern was quasistatic. This factor is calculated using twodimensional spatial filtering; this approach is validated for one vibration source to adequately match a comprehensive AO simulation using MAOS. ${ }^{14}$ At each frequency, the uncorrected and corrected WFEs are computed for two phases of the waveform that are $90 \mathrm{deg}$ apart; the temporal rms over one full period of the sinusoidal waveform can be obtained from the response at these two phases. Taking into account both temporal and spatial effects, the net reduction factor that multiplies the rms uncorrected WFE is $R=1-(1-A)(1-S)$. The spatial fitting error is generally small compared with the temporal fitting error from the limited bandwidth because the spatial pattern of the M1 response is relatively smooth: even at $30 \mathrm{~Hz}$, where the temporal correctability only reduces spatially correctable motion by a factor of two $(S \sim 0.5), 95 \%$ of the motion is spatially correctable $(A \sim 0.05$; Fig. 3 and 4$)$. The length-scales of M1 motion are determined primarily by mirror cell dynamics rather than the specific source (that is, the factor $A$ is largely independent of where forces are applied). This lengthscale is roughly consistent with the length-scales of M1 segment motion inferred from AO DM data at Keck. ${ }^{15}$
The combination of soft voice-coil actuators for M1 and the lower AO-rejection bandwidth for tip/tilt means that the residual AO-corrected WFE is dominated by image jitter at higher frequencies. At lower frequencies, the type II (double-integrator) AO tip/tilt rejection reduces this component, leaving the M1 component relatively more important. This is illustrated in Fig. 5. Above $\sim 10 \mathrm{~Hz}$, the image jitter is typically dominated by motion of M2; overall telescope motion and M1 tip/tilt are important contributors to image jitter below $10 \mathrm{~Hz}$.

The combined AO-corrected WFE per Newton of force is shown in Fig. 6 for the locations described earlier. For each source location other than cable wrap torques and pier forces, the AO-corrected performance is computed for forces in each direction $(x, y, z)$ and the $\mathrm{rms}$ over the three directions shown. A key observation is that the most sensitive frequency range for vibration forces is typically 5 to $20 \mathrm{~Hz}$. At lower frequencies, AO rejection is sufficiently good that the sensitivity decreases rapidly with decreasing frequency; there is also no dynamic amplification from telescope structural resonances below $5 \mathrm{~Hz}$. At sufficiently high frequency, inertial effects similarly result in a rapid decrease in sensitivity with increasing frequency. For simplicity, we thus (1) define a single sensitivity for each source location as the rms WFE per Newton over this 5 to $20 \mathrm{~Hz}$ band and (2) define a frequency-dependent shaping filter that allows for higher forces at higher or lower frequencies. This shaping filter is given by

$$
W(f)= \begin{cases}\left(f / f_{0}\right)^{2} & f \leq f_{0} \\ 1 & f_{0} \leq f \leq f_{1} \\ \left(f / f_{1}\right)^{-2} & f \geq f_{1}\end{cases}
$$

The second-order dependence on frequency at low frequency is reasonable given the type II AO tip/tilt rejection, while the high-frequency second-order dependence is consistent with the inertial response. This filter is shown with the average sensitivity in Fig. 6. The sensitivity is summarized for each source location in Table 1 . Note that the rms WFE is extremely sensitive to several on-telescope locations in particular.

Forces in the facilities building or enclosure result in WFE only through the resulting pier motion, with some additional attenuation of vibration through the soil/pier. Based on data (a)

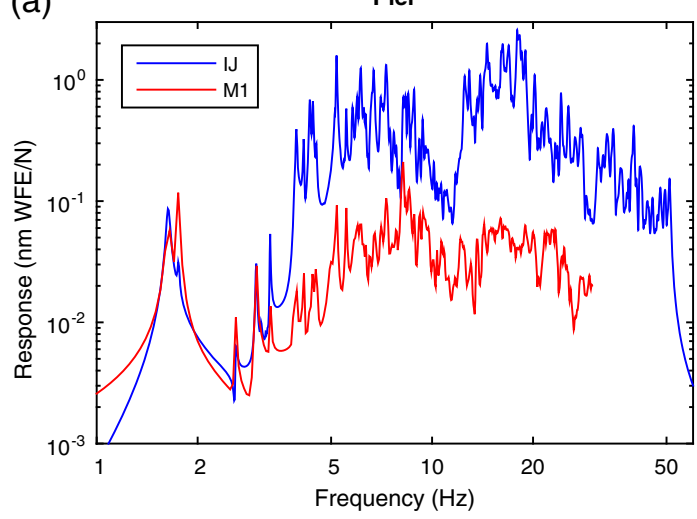

(b)

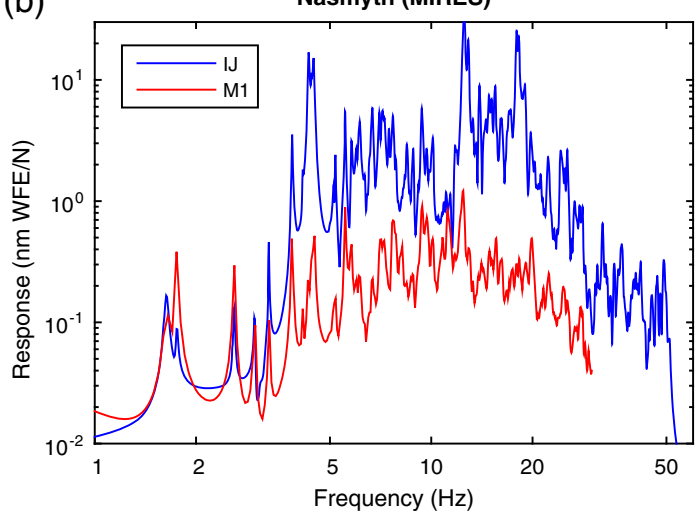

Fig. 5 Contribution to residual AO-corrected WFE from image motion and from M1 segment motion for force input at (a) telescope pier and (b) an instrument. The M1 contribution is typically less important than image jitter, particularly above $10 \mathrm{~Hz}$, due in part to the choice of soft M1CS actuators. The M1 contribution becomes relatively more important at lower frequencies as the image jitter component is reduced by $\mathrm{AO}$ tip/tilt rejection. 

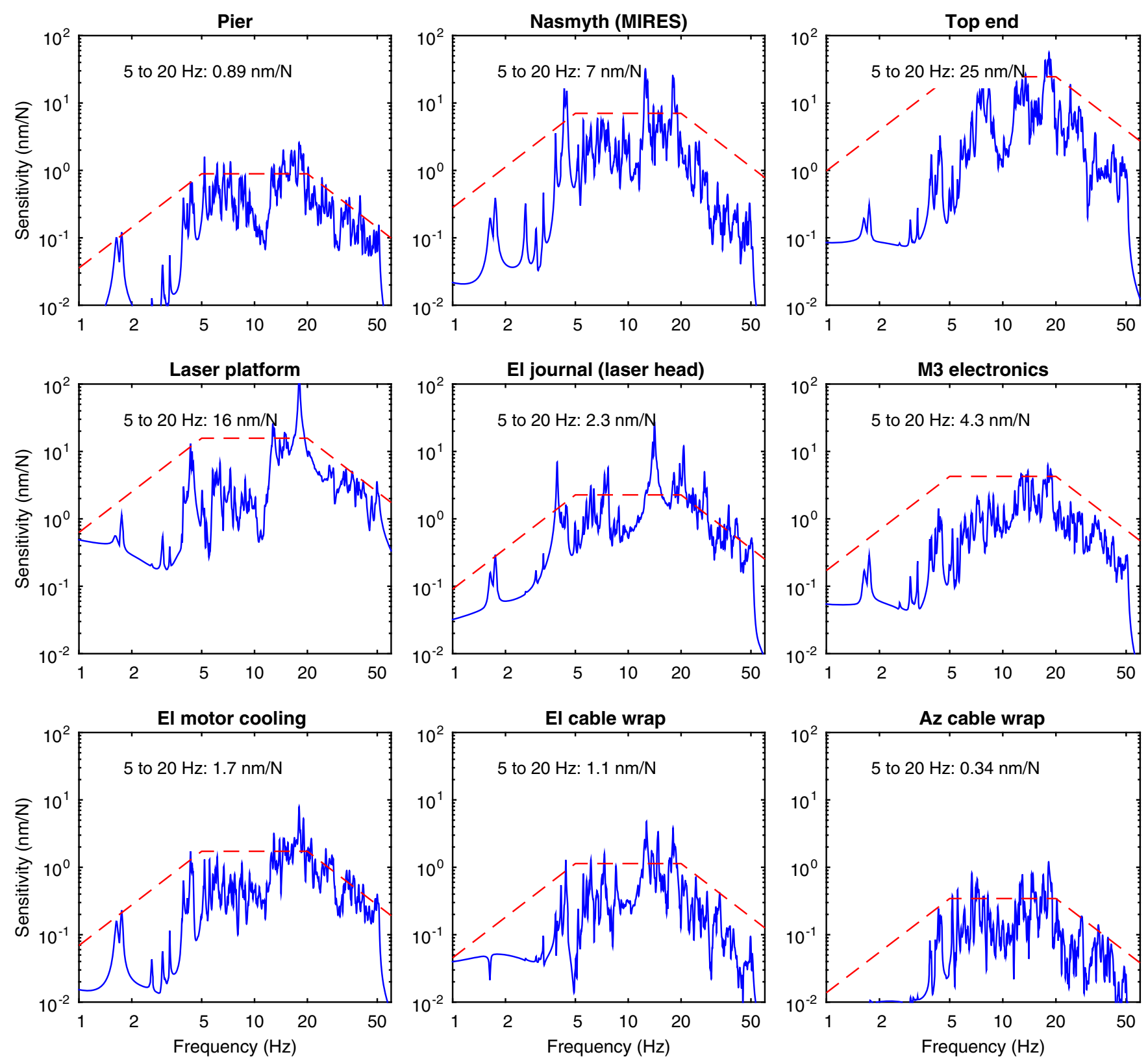

Fig. 6 Sensitivity in nanometers of AO-corrected WFE per Newton of applied force as a function of frequency for forces at the locations given at the beginning of Sec. 2. For each location, the response is computed due to image motion; the contribution due to M1 segment motion is only included for selected locations. With the exception of the pier and cable wrap inputs, the combined WFE plotted is the rms over forces in $x, y$, and $z$ directions. Also shown is the shaping filter used to approximate the sensitivity, and the average sensitivity over the 5 to $20 \mathrm{~Hz}$ range.

collected at Subaru Observatory, we conservatively estimate that a force in the facilities building results in a factor of at least 10 times less response than a force applied at the telescope pier, and a force at the enclosure pier results in a factor of 5 times less response (see Fig. 7 and Ref. 9).

The discussion above focuses on the $\mathrm{AO}$ performance rather than the seeing-limited performance. If force levels from equipment are sufficiently low for the former to be acceptable, then the latter performance will also be acceptable. Sensitivities are also provided in Table 1; any force allocation that meets the AO requirement will yield PSSN of $\sim 0.9999$. A representative PSSN calculation is shown in Fig. 8. Unlike for AO, the M1 contribution is typically larger than the image jitter contribution to PSSN due to the higher spatial frequency content.

\section{Vibration Budget}

The vibration budget is intended to provide an initial force allocation to each subsystem or source of equipment vibration that helps ensure that the overall error budget allocation of equipment vibration is met. Our cost-conscious strategy is to meet performance on average rather than constrain worst-case performance. The vibration budget is constructed from the sensitivities estimated earlier and a subjective estimate of the level of difficulty of meeting force requirements for different sources. This is 
Table 1 Average sensitivity over 5 to $20 \mathrm{~Hz}$ for forces at the locations given in Sec. 2, in nanometers of adaptive optics (AO) corrected wavefront error per Newton of applied force (from Fig. 6), and factor $\beta$ such that normalized point-source sensitivity (PSSN) $=1-\beta^{2} F^{2}$. Sensitivities for sources in facilities building or enclosure pier are estimated as the sensitivity due to forces on the telescope pier, divided by a factor of 10 or 5, respectively (see Fig. 7).

\begin{tabular}{|c|c|c|c|c|c|c|}
\hline \multirow[b]{2}{*}{ Source } & \multicolumn{3}{|c|}{ AO sensitivity $(\mathrm{nm} / \mathrm{N})$} & \multicolumn{3}{|c|}{$\operatorname{PSSN}\left(\mathrm{N}^{-1}\right)$} \\
\hline & IJ & M1 & Combined & $\mathrm{IJ}$ & M1 & Combined \\
\hline Facilities building $^{a}$ & 0.09 & 0.005 & 0.09 & 0.026 & 0.024 & 0.029 \\
\hline Enclosure pier ${ }^{a}$ & 0.18 & 0.01 & 0.18 & 0.05 & 0.05 & 0.058 \\
\hline Telescope pier & 0.89 & 0.05 & 0.89 & 0.26 & 0.24 & 0.29 \\
\hline Instruments and Nasmyth platform & 0.38 & 7.0 & 7.0 & 1.0 & 2.0 & 2.3 \\
\hline Laser platform (laser electronics) & 15.6 & 1.9 & 15.7 & 2.2 & 4.5 & 5.0 \\
\hline El journal (laser head, M2 electronics) & 2.26 & 0.15 & 2.27 & 0.3 & 0.86 & 0.91 \\
\hline Top end (LLT, M2 cell) & 24.5 & 0.66 & 24.5 & 2.6 & 3.1 & 4 \\
\hline M3, M1 electronics & 4.26 & 0.2 & 4.27 & 0.6 & 0.9 & 1.1 \\
\hline Azimuth-axis motor (cooling) & 1.1 & 0.07 & 1.17 & 0.22 & 0.33 & 0.39 \\
\hline Elevation-axis motor (cooling) & 1.72 & 0.1 & 1.73 & 0.22 & 0.51 & 0.56 \\
\hline Azimuth cable wrap & 0.01 & 0.34 & 0.34 & 0.06 & 0.06 & 0.09 \\
\hline Elevation cable wrap & 0.02 & 1.14 & 1.14 & 0.17 & 0.11 & 0.2 \\
\hline
\end{tabular}

${ }^{\mathrm{a}}$ Estimated.

an initial allocation only, which will be revised as further information is available either from design or data.

Our initial allocation is shown in Table 2. For each source, this is the maximum allowable rms force, integrated over all frequencies after passing through the shaping filter described earlier that allows for higher forces at higher or lower frequencies. Individual subsystems can reallocate between subcomponents within their subsystem. While many of the force allocations appear to be exceptionally small, measurements of some representative equipment suggest that with isolation it may be possible to meet these requirements. For example, the cryopump tested in Ref. 9 results in $<0.04 \mathrm{~N} \mathrm{rms} \mathrm{(after} \mathrm{applying}$ the shaping filter) when mounted on $3.6 \mathrm{~Hz}$ isolators.

It is clear that the vibration problem is dominated by components at a few locations with particularly high optical sensitivity, including anything at the telescope top-end near M2, the platform supporting the lasers (this may be amenable to redesign), and the Nasmyth platforms. Some large sources of
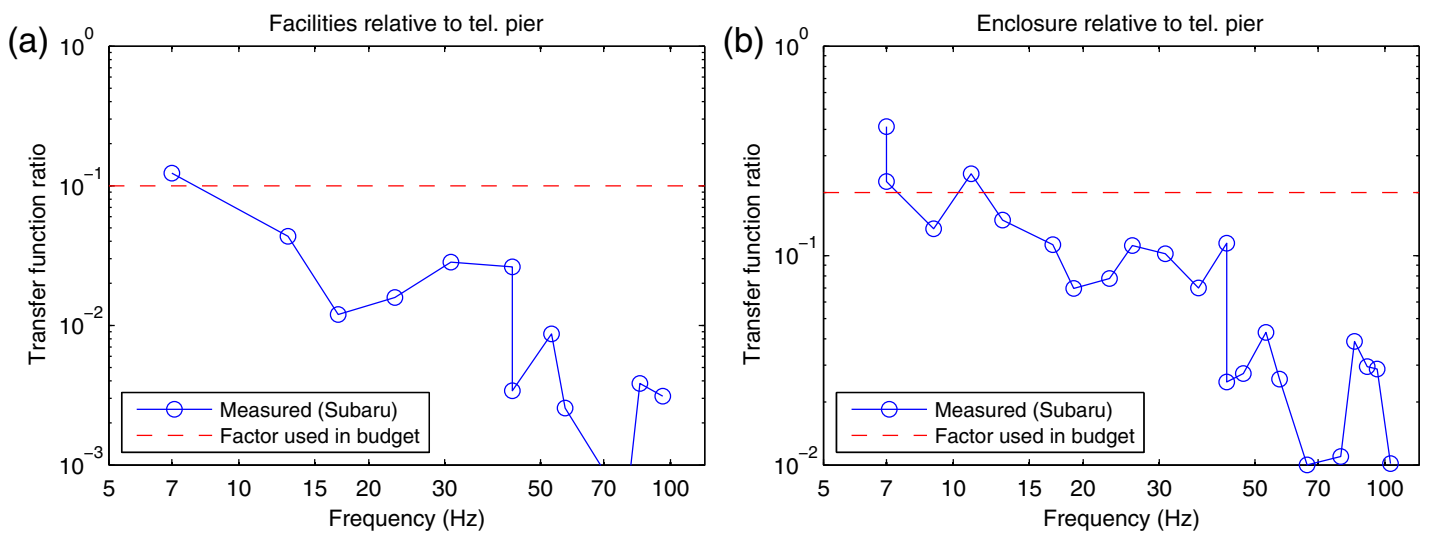

Fig. 7 Ratio of vertical motion on telescope pier due to a force applied on the telescope pier compared with response at the same location due to a force applied in the (a) facilities building or (b) enclosure pier. These are measured at Subaru Observatory, using reciprocity to obtain the transfer functions from remote locations. Reduction factors for horizontal motion are comparable. The reduction factors used in constructing the current TMT budget are likely to be conservative except at very low frequency, although of course the soil and pier characteristics differ between the sites. 

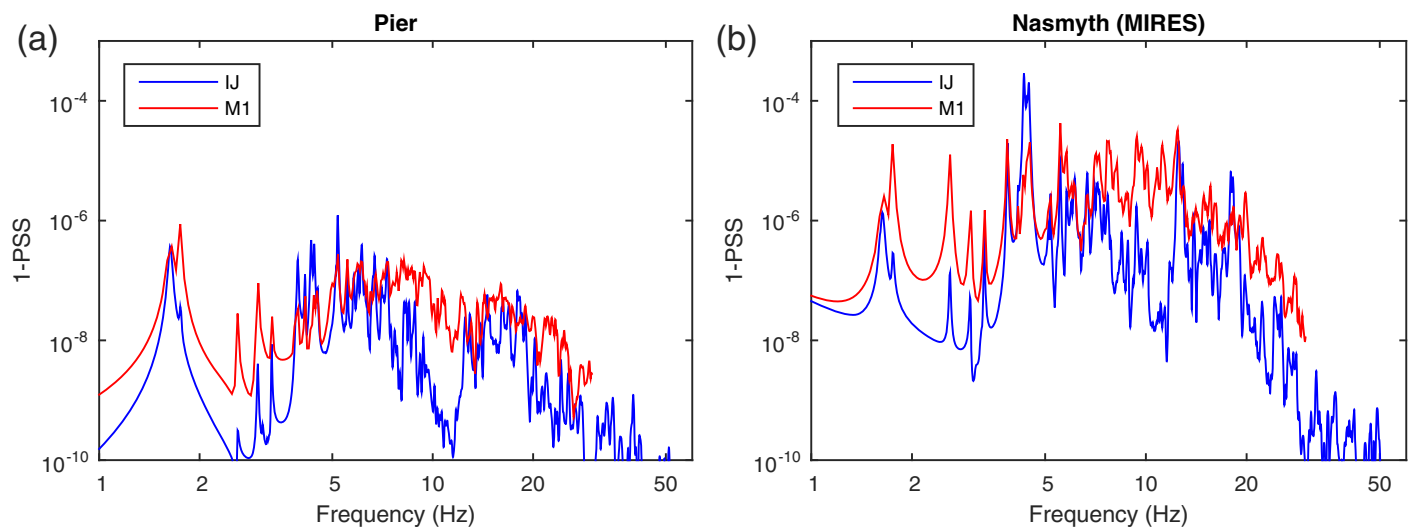

Fig. 8 Contribution to normalized point-source sensitivity (PSSN) from image motion and from M1 segment motion for a $1 \mathrm{~N}$ force input at (a) telescope pier and (b) an instrument; the PSSN contribution scales quadratically with force. The higher spatial frequency M1 contribution is typically more important than image jitter.

Table 2 Preliminary vibration budget, giving the allowable force level for each source, and the estimated sensitivity (Table 1) and corresponding $\mathrm{AO}-$ corrected wavefront error; the aggregate wavefront error from each subsystem is also given. Many subsystems have equipment in the support building; for clarity, these are grouped here under Miscellaneous.

\begin{tabular}{|c|c|c|c|c|c|}
\hline Subsystem & Subcomponent & $\begin{array}{l}\text { Sensitivity } \\
(\mathrm{nm} / \mathrm{N})\end{array}$ & $\begin{array}{l}\text { Allowable force } \\
\text { (N rms) }\end{array}$ & $\begin{array}{l}\text { Subcomponent } \\
\text { AO-WFE }(\mathrm{nm})\end{array}$ & $\begin{array}{c}\text { Aggregate } \\
\text { AO-WFE }(\mathrm{nm})\end{array}$ \\
\hline On telescope & & & & & 23.9 \\
\hline \multirow[t]{8}{*}{ Tel. structure } & & & & & 11.1 \\
\hline & Az drive motor & 1.2 & 1 & 1.2 & \\
\hline & El drive motor & 1.7 & 1 & 1.7 & \\
\hline & Az cable wrap & 0.3 & 1 & 0.3 & \\
\hline & El cable wrap & 1.1 & 1 & 1.1 & \\
\hline & Hydrostatic bearing oil distribution & 0.9 & 1 & 0.9 & \\
\hline & Tel. utility serv. & 7 & 1.4 & 9.9 & \\
\hline & Other & 4.3 & 1 & 4.3 & \\
\hline $\begin{array}{l}\text { Primary mirror } \\
\text { control system }\end{array}$ & & 4.3 & 0.5 & 2.1 & 2.1 \\
\hline \multirow[t]{3}{*}{ M2 system } & & & & & 5.0 \\
\hline & M2 cell & 24.5 & 0.2 & 4.9 & \\
\hline & M2 electronics & 2.3 & 0.5 & 1.1 & \\
\hline M3 system & & 4.3 & 0.5 & 2.1 & 2.1 \\
\hline Comm. \& info. systems & & 7.0 & 0.5 & 3.5 & 3.5 \\
\hline \multirow[t]{5}{*}{ Laser guide-star facility } & & & & & 10.0 \\
\hline & Top-end & 24.50 & 0.2 & 4.9 & \\
\hline & Beam-transfer optics & 7.0 & 0.5 & 3.5 & \\
\hline & Lasers & 2.3 & 0.5 & 1.1 & \\
\hline & Laser electronics & 15.7 & 0.5 & 7.9 & \\
\hline
\end{tabular}


Table 2 (Continued).

\begin{tabular}{|c|c|c|c|c|c|}
\hline Subsystem & Subcomponent & $\begin{array}{l}\text { Sensitivity } \\
(\mathrm{nm} / \mathrm{N})\end{array}$ & $\begin{array}{l}\text { Allowable force } \\
\text { (N rms) }\end{array}$ & $\begin{array}{l}\text { Subcomponent } \\
\text { AO-WFE }(\mathrm{nm})\end{array}$ & $\begin{array}{c}\text { Aggregate } \\
\text { AO-WFE }(\mathrm{nm})\end{array}$ \\
\hline \multirow[t]{3}{*}{ Instrumentation cooling } & & & & & 9.9 \\
\hline & Cryocooling & 7.0 & 1 & 7.0 & \\
\hline & Refrigerant cooling & 7.0 & 1 & 7.0 & \\
\hline \multirow[t]{12}{*}{ Instruments } & & & & & 14.2 \\
\hline & NFIRAOS & 7.0 & 1 & 7.0 & \\
\hline & IRIS & 7.0 & 0.5 & 3.5 & \\
\hline & WFOS & 7.0 & 0.8 & 5.6 & \\
\hline & IRMS/MOSFIRE & 7.0 & 0.5 & 3.5 & \\
\hline & HROS & 7.0 & 0.5 & 3.5 & \\
\hline & IRMOS & 7.0 & 0.5 & 3.5 & \\
\hline & PFI & 7.0 & 0.5 & 3.5 & \\
\hline & MIRAO & 7.0 & 0.5 & 3.5 & \\
\hline & NIRES-B & 7.0 & 0.5 & 3.5 & \\
\hline & NIRES-R & 7.0 & 0.5 & 3.5 & \\
\hline & WIRC & 7.0 & 0.5 & 3.5 & \\
\hline Within enclosure & & & & & 13.2 \\
\hline Enclosure & & 0.18 & 50 & 8.9 & \\
\hline Summit facilities & & 0.89 & 10 & 8.9 & \\
\hline Tel. structure & & 0.18 & 10 & 1.8 & \\
\hline Optical cleaning sys. & & 0.18 & 10 & 1.8 & \\
\hline Optical coating sys. & & 0.18 & 10 & 1.8 & \\
\hline Test instruments & & 0.18 & 1 & 0.18 & \\
\hline Optics handling eq. & & 0.18 & 10 & 1.8 & \\
\hline Test instrument ctrl & & 0.18 & 1 & 0.18 & \\
\hline Observatory safety sys. & & 0.18 & 1 & 0.18 & \\
\hline Engineering sensors & & 0.18 & 10 & 1.8 & \\
\hline Comm. \& info. sys & & 0.18 & 1 & 0.18 & \\
\hline Inside support building & & & & & 10.8 \\
\hline Summit facilities & & 0.09 & 100 & 8.9 & \\
\hline Telescope structure & & 0.09 & 50 & 4.5 & \\
\hline Miscellaneous & & 0.09 & 50 & 4.5 & \\
\hline
\end{tabular}

Note: WFE, wavefront error.

vibration are located in the summit support building (e.g., chillers and pumps associated with the Summit Facilities subsystem, and hydrostatic bearing pumps associated with the telescope structure subsystem). However, because forces in these locations are significantly attenuated through the soil, relatively larger force allocations are allowable.

\section{Conclusions}

Image degradation due to equipment vibration has typically been addressed solely through best practices design choices, such as selecting low-vibration equipment, mounting equipment off-telescope where practical, and isolating large vibration sources. While these steps are clearly essential, TMT is 
establishing requirements on the allowable force levels at different locations in order to ensure that the AO-corrected WFEs induced by vibrating equipment remain at acceptable levels and to provide a framework for making cost-effective design choices. This vibration budget is based on the modeled optical sensitivity resulting from vibrating forces at different locations and different frequencies.

Note that our sensitivities describe an average expected response to vibration and not the worst case that might arise if a vibration source aligns with a structural resonance; bounding the worst-case performance would require substantially tighter force requirements but could follow similar methodology.

There is clearly substantial uncertainty in predicting the sensitivity of rms WFE to forces as derived herein; the expected force level from any particular source is also uncertain. Modeling the response of the telescope structure at the frequencies relevant for equipment vibration is challenging and not likely to be highly accurate. Furthermore, it is difficult to estimate unsteady forces from any equipment sources, particularly early in the design. However, given the importance of vibration in AO observations, it is essential that we make the best effort possible with the existing tools. We hope that this effort will greatly increase the probability of delivering an observatory without significant vibration-related WFEs. We present this tool with the hope that this approach may be a model for other observatories as well.

\section{Acknowledgments}

Daigo Tomono and Hiroshi Terada of NAOJ/Subaru and Peter Byrnes of HIA, NRC were instrumental in collecting the data in Fig. 7. The TMT Project gratefully acknowledges the support of the TMT collaborating institutions. They are the Association of Canadian Universities for Research in Astronomy, the California Institute of Technology, the University of California, the National Astronomical Observatory of Japan, the National Astronomical Observatories of China and their consortium partners, and the Department of Science and Technology of India and their supported institutes. This work was supported as well by the Gordon and Betty Moore Foundation, the Canada Foundation for Innovation, the Ontario Ministry of Research and Innovation, the National Research Council of Canada, the Natural Sciences and Engineering Research Council of Canada, the British Columbia Knowledge Development Fund, the Association of Universities for Research in Astronomy, the U.S. National Science Foundation, and the National Institutes of Natural Sciences of Japan.

\section{References}

1. C. Kulcsár et al., "Vibrations in AO control: a short analysis of on-sky data around the world," Proc. SPIE 8447, 84471C (2012).

2. B. H. Thomas, W. Neville, and J. Snodgrass, "Assessment and control of vibrations affecting large astronomical telescopes," Proc. SPIE 0732, 130-156 (1987).

3. C. Correia, J. P. Véran, and G. Herriot, "Advanced vibration suppression algorithms in adaptive optics systems," J. Opt. Soc. Am. A 29(3), 185-194 (2012).

4. A. Guesalaga et al., "Comparison of vibration mitigation controllers for adaptive optics systems," Appl. Opt. 51(19), 4520-4535 (2012).

5. C. Kulcsár et al., "Vibration mitigation in adaptive optics control," Proc. SPIE 8447, 84470Z (2012).

6. M. Böhm et al., "Real-time vibration compensation for large telescopes," Monthly Notices R. Astron. Soc. 442, 2466-2455 (2014).

7. E. H. Anderson, R. M. Glaese, and D. Neill, "A comparison of vibration damping methods for ground based telescopes," Proc. SPIE 7012, 70120H (2008).

8. M. Hess et al., "Strategy for identifying and mitigating facility vibrations to improve optical performance at the W. M. Keck Observatory," Proc. SPIE 4837, 342-351 (2003).

9. H. Thompson et al., "Measuring transmission and forces from observatory equipment vibration," Proc. SPIE 9145, 91454G (2014).

10. D. G. MacMartin and H. Thompson, "Development of vibration source requirements for TMT to ensure AO performance," 2013, http://ao4elt3. arcetri.astro.it/proceedings/fulltext_13362.pdf (7 August 2015).

11. D. G. MacMartin and H. Thompson, "Equipment vibration budget for TMT," Proc. SPIE 9145, $91452 \mathrm{O}$ (2014).

12. H. Lamb, "On the propagation of tremors over the surface of an elastic solid," Philos. Trans. R. Soc. A 203, 1-42 (1904).

13. D. G. MacMartin et al., "Dynamic analysis of the active-controlled segmented mirror of the Thirty Meter Telescope," IEEE Trans. Control Syst. Technol. 22(1), 58-68 (2014).

14. L. Wang and B. Ellerbroek, "Computer simulations and real-time control of ELT AO systems using graphical processing units," Proc. SPIE 8447, 844723 (2012).

15. D. G. MacMynowski et al., "Primary mirror dynamic disturbance models for TMT: vibration and wind," Proc. SPIE 7738, 77380E (2010).

Douglas G. MacMartin is a research professor in computing and mathematical sciences at the California Institute of Technology. Prior to 2000 he led the active control "theme" at United Technologies Research Center. He received his PhD from MIT in aeronautics and astronautics in 1992 and his BASc in engineering science from the University of Toronto in 1987. In addition to telescope dynamics and control for TMT, his research interests include climate dynamics and geoengineering.

Hugh Thompson has a degree in engineering physics from the University of British Columbia. Prior to joining the Thirty Meter Telescope Project as a senior systems engineer in 2008 he was a spacecraft systems engineer at MDA. There he was responsible for the thermal system of the RADARSAT-2 satellite and was later the optical payload systems engineer for the Rapid Eye constellation of five satellites. He works at NRC Herzberg in Victoria, Canada. 